\title{
THE USE OF ELECTRE I METHOD IN THE DECISION-MAKING PROCESS - CASE STUDY
}

\author{
Zygmunt KORBAN ${ }^{1}$, Zdeněk BOHÁĆ
}

Review article

This article presents the possibilities of using one of the multi-criteria discrete methods
in the engineering design. Illustrated with an example, the way of using Electre I method
in the evaluation of quality features, which describe technical means the purchase of
which is being taken into consideration in connection with the expected start-up of a new
coal-mine area, has been discussed.
Keywords: $\quad$ Statistical methods, Discrete methods, Multi-criteria methods, Decision theory, Electre I.

\section{Introduction}

In the selection of technical and organisational system, apart from single-criteria evaluations (the evaluation criterion is, for example, the limit payback period), more and more often the multi-criteria evaluations are also used. The example of the latter ones includes, but is not limited to, the distance measure formulas and multi-criteria discrete methods (Mynarski, 1992; Trzaskalik, 2006). For methods using the distance measure formulas there can be distinguished, among other things, the Hamming, Euclidean, Bray-Curtis, JeffreysMatusita, Clark and Canberra distances, while for multi-criteria discrete methods - the Analytic Hierarchy Process (AHP), Promethee II and Electre I methods. The last method allows the set of $n$ variants of decision to be analysed and evaluated with regard to $k$ maximised $f_{k}$ criteria - each individual variant is assigned by the decision maker the importance $w_{k}\left(\sum_{i=1}^{k} w_{i}=1\right) \quad($ Hillier and Lieberman, 1990; Trzaskalik, 2001; Wagner, 1980). With two variants of decision $x$ and $y$, the decision maker determines the outranking index $\varphi_{i}(x, y)$ :

$$
\varphi_{i}(x, y)= \begin{cases}1, & \text { when } f_{i}(x) \geq f_{i}(y) \\ 0, & \text { otherwise }\end{cases}
$$

which determines whether variant $x$ is evaluated better or worse than variant $y$ with regard to consecutive $f_{k}$ criteria. Then, in order to determine the degree of predominance of variant $x$ over variant $y$, the concordance index $c(x, y)$ is calculated:

$$
c(x, y)=\sum_{i=1}^{k} w_{i} \varphi_{i}(x, y)
$$

which is the sum of weights of these criteria for which the values of variant $x$ are not smaller than those for variant $y$. The concordance condition is assumed to be met when the concordance index is not lower than the indifference threshold value adopted by the decision maker $(s)$ :

$$
c(x, y) \geq s, \quad s \in\langle 0.5 ; 1.0\rangle
$$

In order to eliminate these cases where the concordance condition is admittedly met, but at least one of the criteria of predominating variant $x$ has unfavourable value, the lack of concordance condition is checked (the decision maker adopts subjectively the so-called veto threshold value).

The decision-making procedure using Electre I method ends with outlining the graph for relationship between variants of decision (Hillier and Lieberman, 1990; Trzaskalik, 2006; Trzaskalik, 2001; Wagner, 1980).

\section{Materials and Methods}

\section{The example of the use of Electre I method in the decision-making process related to purchase of the means of transport}

Start-up of exploitation in a new coal-mine area is expected, therefore the purchase of the means of

Silesian University of Technology, Faculty of Mining and Geology, Gliwice, Poland, zygmunt.korban@polsl.pl

2 VŠB - Technical University of Ostrava, Department of Mathematics and Descriptive Geometry, Ostrava, Czech Republic, zdenek.bohac@vsb.cz 
transport for carrying people and materials to the said area is being considered. As a part of the initial evaluation (after technical parameters of the transport machinery and equipment were analysed and initial calculation of the costs related to the purchase/ use of individual variants of technical equipment was carried out), three alternatives were selected: purchase of the cog-wheel railway (variant $a^{1}$ ), purchase of the suspended diesel railway (variant $a^{2}$ ) and purchase of the suspended railway with rope drive (variant $a^{3}$ ). In addition, six evaluation criteria were defined: ease of assembly $\left(f_{1}\right)$; availability of spare parts $\left(f_{2}\right)$; safety $\left(f_{3}\right)$; potential for expansion $\left(f_{4}\right)$; terms and conditions of warranty and service $\left(f_{5}\right)$ and manufacturer's brand $\left(f_{6}\right)$.

After survey research in the group of experts (employees of the materials management department, supervisory personnel of the electrical power and mechanical department and employees of the underground transport department), the consecutive criteria were assigned the importance coefficients:

$$
\begin{aligned}
& w_{1}=0.3 \\
& w_{2}=0.2 \\
& w_{3}=0.2 \\
& w_{4}=0.1 \\
& w_{5}=0.1 \\
& w_{6}=0.1
\end{aligned}
$$

whereas $\sum_{i=1}^{6} w_{i}=1$

Tab. 1 presents the evaluations of variants taken into consideration with regard to the defined criteria (the evaluation scale for each of the criteria from 0 to 10$)$.

Tab. 1 Evaluation of equipment variants with regard to the adopted criteria

\begin{tabular}{|c|c|c|c|c|c|c|c|}
\cline { 3 - 9 } \multicolumn{2}{c|}{} & \multicolumn{7}{|c|}{ Criterion } \\
\cline { 3 - 9 } & $\mathbf{f}_{\mathbf{1}}$ & $\mathbf{f}_{\mathbf{2}}$ & $\mathbf{f}_{\mathbf{3}}$ & $\mathbf{f}_{\mathbf{4}}$ & $\mathbf{f}_{\mathbf{5}}$ & $\mathbf{f}_{\mathbf{6}}$ \\
\hline \multirow{3}{*}{ Variants } & $\mathbf{a}_{\mathbf{1}}$ & 7 & 7 & 10 & 3 & 4 & 2 \\
\cline { 2 - 9 } & $\mathbf{a}_{\mathbf{2}}$ & 3 & 9 & 10 & 1 & 7 & 9 \\
\cline { 2 - 9 } & $\mathbf{a}_{\mathbf{3}}$ & 3 & 6 & 10 & 8 & 6 & 3 \\
\hline
\end{tabular}

For consecutive criteria the following veto thresholds were adopted:

$$
\begin{array}{ll}
v_{1}\left[f_{1}\left(a^{i}\right)\right]=4 & v_{4}\left[f_{4}\left(a^{i}\right)\right]=7 \\
v_{2}\left[f_{2}\left(a^{i}\right)\right]=6 & v_{5}\left[f_{5}\left(a^{i}\right)\right]=6 \\
v_{3}\left[f_{3}\left(a^{i}\right)\right]=6 & v_{6}\left[f_{6}\left(a^{i}\right)\right]=2
\end{array}
$$

At the same time, the indifference threshold of $0.6(s=0.6)$ was adopted.
In the first stage, the set of concordances was determined - for $k \in\langle 1 ; 6\rangle$ of possible pairs of variants of decision and the values of $\varphi_{k}\left(a^{i}, a^{j}\right)$ were calculated. The results are summarised in Tab. 2.

Tab. 2 Sets of concordances for pairs of variants

\begin{tabular}{|c|c|c|c|}
\hline $\mathbf{\Phi}_{\mathbf{1}}$ & $\mathbf{a}^{\mathbf{1}}$ & $\mathbf{a}^{\mathbf{2}}$ & $\mathbf{a}^{\mathbf{3}}$ \\
\hline $\mathbf{a}^{\mathbf{1}}$ & 1 & 1 & 1 \\
\hline $\mathbf{a}^{\mathbf{2}}$ & 0 & 1 & 1 \\
\hline $\mathbf{a}^{\mathbf{3}}$ & 0 & 1 & 1 \\
\hline
\end{tabular}

\begin{tabular}{|c|c|c|c|}
\hline $\mathbf{\Phi}_{2}$ & $\mathbf{a}^{\mathbf{1}}$ & $\mathbf{a}^{\mathbf{2}}$ & $\mathbf{a}^{\mathbf{3}}$ \\
\hline $\mathbf{a}^{\mathbf{1}}$ & 1 & 0 & 1 \\
\hline $\mathbf{a}^{\mathbf{2}}$ & 1 & 1 & 1 \\
\hline $\mathbf{a}^{\mathbf{3}}$ & 0 & 0 & 1 \\
\hline
\end{tabular}

\begin{tabular}{|c|c|c|c|}
\hline $\mathbf{\Phi}_{\mathbf{3}}$ & $\mathbf{a}^{\mathbf{1}}$ & $\mathbf{a}^{\mathbf{2}}$ & $\mathbf{a}^{\mathbf{3}}$ \\
\hline $\mathbf{a}^{\mathbf{1}}$ & 1 & 1 & 1 \\
\hline $\mathbf{a}^{\mathbf{2}}$ & 1 & 1 & 1 \\
\hline $\mathbf{a}^{\mathbf{3}}$ & 1 & 1 & 1 \\
\hline
\end{tabular}

\begin{tabular}{|c|c|c|c|}
\hline$\Phi_{4}$ & $\mathbf{a}^{\mathbf{1}}$ & $\mathbf{a}^{\mathbf{2}}$ & $\mathbf{a}^{\mathbf{3}}$ \\
\hline $\mathbf{a}^{\mathbf{1}}$ & 1 & 1 & 0 \\
\hline $\mathbf{a}^{\mathbf{2}}$ & 0 & 1 & 0 \\
\hline $\mathbf{a}^{\mathbf{3}}$ & 1 & 1 & 1 \\
\hline
\end{tabular}

\begin{tabular}{|c|c|c|c|}
\hline $\boldsymbol{\Phi}_{\mathbf{5}}$ & $\mathbf{a}^{\mathbf{1}}$ & $\mathbf{a}^{\mathbf{2}}$ & $\mathbf{a}^{\mathbf{3}}$ \\
\hline $\mathbf{a}^{\mathbf{1}}$ & 1 & 0 & 0 \\
\hline $\mathbf{a}^{\mathbf{2}}$ & 1 & 1 & 1 \\
\hline $\mathbf{a}^{\mathbf{3}}$ & 1 & 0 & 1 \\
\hline
\end{tabular}

\begin{tabular}{|c|c|c|c|}
\hline $\mathbf{\Phi}_{\mathbf{6}}$ & $\mathbf{a}^{\mathbf{1}}$ & $\mathbf{a}^{\mathbf{2}}$ & $\mathbf{a}^{\mathbf{3}}$ \\
\hline $\mathbf{a}^{\mathbf{1}}$ & 1 & 0 & 0 \\
\hline $\mathbf{a}^{\mathbf{2}}$ & 1 & 1 & 1 \\
\hline $\mathbf{a}^{\mathbf{3}}$ & 1 & 0 & 1 \\
\hline
\end{tabular}

Based on the adopted importance coefficients $w_{k}$ and values of $\varphi_{k}\left(a^{i}, a^{j}\right)$, the concordance indices were determined:

$$
\begin{gathered}
c\left(a^{1}, a^{2}\right)=w_{1} \varphi_{1}\left(a^{1}, a^{2}\right)+w_{2} \varphi_{2}\left(a^{1}, a^{2}\right)+ \\
+w_{3} \varphi_{3}\left(a^{1}, a^{2}\right)+w_{4} \varphi_{4}\left(a^{1}, a^{2}\right)+ \\
+w_{5} \varphi_{5}\left(a^{1}, a^{2}\right)+w_{6} \varphi_{6}\left(a^{1}, a^{2}\right)=0.6
\end{gathered}
$$

$$
\begin{gathered}
c\left(a^{1}, a^{3}\right)=w_{1} \varphi_{1}\left(a^{1}, a^{3}\right)+w_{2} \varphi_{2}\left(a^{1}, a^{3}\right)+ \\
+w_{3} \varphi_{3}\left(a^{1}, a^{3}\right)+w_{4} \varphi_{4}\left(a^{1}, a^{3}\right)+ \\
+w_{5} \varphi_{5}\left(a^{1}, a^{3}\right)+w_{6} \varphi_{6}\left(a^{1}, a^{3}\right)=0.7
\end{gathered}
$$

$$
\begin{gathered}
c\left(a^{2}, a^{1}\right)=w_{1} \varphi_{1}\left(a^{2}, a^{1}\right)+w_{2} \varphi_{2}\left(a^{2}, a^{1}\right)+ \\
+w_{3} \varphi_{3}\left(a^{2}, a^{1}\right)+w_{4} \varphi_{4}\left(a^{2}, a^{1}\right)+ \\
+w_{5} \varphi_{5}\left(a^{2}, a^{1}\right)+w_{6} \varphi_{6}\left(a^{2}, a^{1}\right)=0.6
\end{gathered}
$$

$$
\begin{gathered}
c\left(a^{2}, a^{3}\right)=w_{1} \varphi_{1}\left(a^{2}, a^{3}\right)+w_{2} \varphi_{2}\left(a^{2}, a^{3}\right)+ \\
+w_{3} \varphi_{3}\left(a^{2}, a^{3}\right)+w_{4} \varphi_{4}\left(a^{2}, a^{3}\right)+ \\
+w_{5} \varphi_{5}\left(a^{2}, a^{3}\right)+w_{6} \varphi_{6}\left(a^{2}, a^{3}\right)=0.9
\end{gathered}
$$

$$
\begin{gathered}
c\left(a^{3}, a^{1}\right)=w_{1} \varphi_{1}\left(a^{3}, a^{1}\right)+w_{2} \varphi_{2}\left(a^{3}, a^{1}\right)+ \\
+w_{3} \varphi_{3}\left(a^{3}, a^{1}\right)+w_{4} \varphi_{4}\left(a^{3}, a^{1}\right)+ \\
+w_{5} \varphi_{5}\left(a^{3}, a^{1}\right)+w_{6} \varphi_{6}\left(a^{3}, a^{1}\right)=0.5
\end{gathered}
$$




$$
\begin{gathered}
c\left(a^{3}, a^{2}\right)=w_{1} \varphi_{1}\left(a^{3}, a^{2}\right)+w_{2} \varphi_{2}\left(a^{3}, a^{2}\right)+ \\
+w_{3} \varphi_{3}\left(a^{3}, a^{2}\right)+w_{4} \varphi_{4}\left(a^{3}, a^{2}\right)+ \\
+w_{5} \varphi_{5}\left(a^{3}, a^{2}\right)+w_{6} \varphi_{6}\left(a^{3}, a^{2}\right)=0.6
\end{gathered}
$$

The results of calculation are summarised in Tab. 3 .

Tab. 3 Results of calculation

\begin{tabular}{|c|c|c|c|}
\hline $\mathbf{C}$ & $\mathbf{a}^{\mathbf{1}}$ & $\mathbf{a}^{\mathbf{2}}$ & $\mathbf{a}^{\mathbf{3}}$ \\
\hline $\mathbf{a}^{\mathbf{1}}$ & 1 & 0.6 & 0.7 \\
\hline $\mathbf{a}^{\mathbf{2}}$ & 0.6 & 1 & 0.9 \\
\hline $\mathbf{a}^{\mathbf{3}}$ & 0.5 & 0.6 & 1 \\
\hline
\end{tabular}

The set of concordances in the binary form (for the adopted indifference threshold $s=0.6$ ) is presented in Tab. 4.

Tab. 4 Set of concordances in the form of binary matrix

\begin{tabular}{|c|c|c|c|}
\hline $\mathbf{C}$ & $\mathbf{a}^{\mathbf{1}}$ & $\mathbf{a}^{\mathbf{2}}$ & $\mathbf{a}^{\mathbf{3}}$ \\
\hline $\mathbf{a}^{\mathbf{1}}$ & 1 & 1 & 1 \\
\hline $\mathbf{a}^{\mathbf{2}}$ & 1 & 1 & 1 \\
\hline $\mathbf{a}^{\mathbf{3}}$ & 0 & 1 & 1 \\
\hline
\end{tabular}

\section{Results}

For pairs of variants for which the concordance condition has been met, the lack of disconcordance condition was checked then:

for $k=1$

$$
\begin{array}{r}
\left(a^{1}, a^{2}\right): f_{1}\left(a^{1}\right)+v_{1}\left[f_{1}\left(a^{1}\right)\right] \geq f_{1}\left(a^{2}\right) \\
\text { no disconcordance }
\end{array}
$$

$$
\left(a^{1}, a^{3}\right): f_{1}\left(a^{1}\right)+v_{1}\left[f_{1}\left(a^{1}\right)\right] \geq f_{1}\left(a^{3}\right)
$$

no disconcordance

$$
\begin{array}{r}
\left(a^{2}, a^{1}\right): f_{1}\left(a^{2}\right)+v_{1}\left[f_{1}\left(a^{2}\right)\right] \geq f_{1}\left(a^{1}\right) \\
\text { no disconcordance }
\end{array}
$$

$$
\begin{array}{r}
\left(a^{2}, a^{3}\right): f_{1}\left(a^{2}\right)+v_{1}\left[f_{1}\left(a^{2}\right)\right] \geq f_{1}\left(a^{3}\right) \\
\text { no disconcordance }
\end{array}
$$

$$
\left(a^{3}, a^{2}\right): f_{1}\left(a^{3}\right)+v_{1}\left[f_{1}\left(a^{3}\right)\right] \geq f_{1}\left(a^{2}\right)
$$

$$
\begin{array}{r}
\left(a^{1}, a^{3}\right): f_{2}\left(a^{1}\right)+v_{2}\left[f_{2}\left(a^{1}\right)\right] \geq f_{2}\left(a^{3}\right) \\
\text { no disconcordance } \\
\left(a^{2}, a^{1}\right): f_{2}\left(a^{2}\right)+v_{2}\left[f_{2}\left(a^{2}\right)\right] \geq f_{2}\left(a^{1}\right)
\end{array}
$$
no disconcordance

$$
\left(a^{2}, a^{3}\right): f_{2}\left(a^{2}\right)+v_{2}\left[f_{2}\left(a^{2}\right)\right] \geq f_{2}\left(a^{3}\right)
$$
no disconcordance

$$
\left(a^{3}, a^{2}\right): f_{2}\left(a^{3}\right)+v_{2}\left[f_{2}\left(a^{3}\right)\right] \geq f_{2}\left(a^{2}\right)
$$

no disconcordance

$$
\text { for } k=3
$$

$$
\left(a^{1}, a^{2}\right): f_{3}\left(a^{1}\right)+v_{3}\left[f_{3}\left(a^{1}\right)\right] \geq f_{3}\left(a^{2}\right)
$$

no disconcordance

$$
\left(a^{1}, a^{3}\right): f_{3}\left(a^{1}\right)+v_{3}\left[f_{3}\left(a^{1}\right)\right] \geq f_{3}\left(a^{3}\right)
$$
no disconcordance

$$
\begin{array}{r}
\left(a^{2}, a^{1}\right): f_{3}\left(a^{2}\right)+v_{3}\left[f_{3}\left(a^{2}\right)\right] \geq f_{3}\left(a^{1}\right) \\
\text { no disconcordance }
\end{array}
$$

$$
\left(a^{2}, a^{3}\right): f_{3}\left(a^{2}\right)+v_{3}\left[f_{3}\left(a^{2}\right)\right] \geq f_{3}\left(a^{3}\right)
$$
no disconcordance

$$
\left(a^{3}, a^{2}\right): f_{3}\left(a^{3}\right)+v_{3}\left[f_{3}\left(a^{3}\right)\right] \geq f_{3}\left(a^{2}\right)
$$
no disconcordance

$$
\text { for } k=4
$$

$$
\left(a^{1}, a^{2}\right): f_{4}\left(a^{1}\right)+v_{4}\left[f_{4}\left(a^{1}\right)\right] \geq f_{4}\left(a^{2}\right)
$$
no disconcordance

$$
\left(a^{1}, a^{3}\right): f_{4}\left(a^{1}\right)+v_{4}\left[f_{4}\left(a^{1}\right)\right] \geq f_{4}\left(a^{3}\right)
$$
no disconcordance

$$
\left(a^{2}, a^{1}\right): f_{4}\left(a^{2}\right)+v_{4}\left[f_{4}\left(a^{2}\right)\right] \geq f_{4}\left(a^{1}\right)
$$
no disconcordance

$$
\left(a^{2}, a^{3}\right): f_{4}\left(a^{2}\right)+v_{4}\left[f_{4}\left(a^{2}\right)\right] \geq f_{4}\left(a^{3}\right)
$$
no disconcordance

$$
\begin{array}{r}
\left(a^{3}, a^{2}\right): f_{4}\left(a^{3}\right)+v_{4}\left[f_{4}\left(a^{3}\right)\right] \geq f_{4}\left(a^{2}\right) \\
\text { no disconcordance }
\end{array}
$$

$$
\text { for } k=5
$$

$$
\left(a^{1}, a^{2}\right): f_{5}\left(a^{1}\right)+v_{5}\left[f_{5}\left(a^{1}\right)\right] \geq f_{5}\left(a^{2}\right)
$$

\footnotetext{
$\left(a^{1}, a^{2}\right): f_{2}\left(a^{1}\right)+v_{2}\left[f_{2}\left(a^{1}\right)\right] \geq f_{2}\left(a^{2}\right) \quad(7+6 \geq 9)$ no disconcordance
} 


$$
\left(a^{1}, a^{3}\right): f_{5}\left(a^{1}\right)+v_{5}\left[f_{5}\left(a^{1}\right)\right] \geq f_{5}\left(a^{3}\right)
$$

$$
\text { no disconcordance }
$$

$$
\left(a^{2}, a^{1}\right): f_{5}\left(a^{2}\right)+v_{5}\left[f_{5}\left(a^{2}\right)\right] \geq f_{5}\left(a^{1}\right)
$$

no disconcordance

$$
\left(a^{2}, a^{3}\right): f_{5}\left(a^{2}\right)+v_{5}\left[f_{5}\left(a^{2}\right)\right] \geq f_{5}\left(a^{3}\right)
$$

no disconcordance

$$
\left(a^{3}, a^{2}\right): f_{5}\left(a^{3}\right)+v_{5}\left[f_{5}\left(a^{3}\right)\right] \geq f_{5}\left(a^{2}\right)
$$

$$
\text { no disconcordance }
$$

for $k=6$

$$
\begin{array}{r}
\left(a^{1}, a^{2}\right): f_{6}\left(a^{1}\right)+v_{6}\left[f_{6}\left(a^{1}\right)\right] \geq f_{6}\left(a^{2}\right) \\
\text { no disconcordance } \\
\left(a^{1}, a^{3}\right): f_{6}\left(a^{1}\right)+v_{6}\left[f_{6}\left(a^{1}\right)\right] \geq f_{6}\left(a^{3}\right) \\
\text { no disconcordance } \\
\left(a^{2}, a^{1}\right): f_{6}\left(a^{2}\right)+v_{6}\left[f_{6}\left(a^{2}\right)\right] \geq f_{6}\left(a^{1}\right)
\end{array}
$$$$
\text { no disconcordance }
$$

$$
\left(a^{2}, a^{3}\right): f_{6}\left(a^{2}\right)+v_{6}\left[f_{6}\left(a^{2}\right)\right] \geq f_{6}\left(a^{3}\right)
$$

no disconcordance

$$
\begin{array}{r}
\left(a^{3}, a^{2}\right): f_{6}\left(a^{3}\right)+v_{6}\left[f_{6}\left(a^{3}\right)\right] \geq f_{6}\left(a^{2}\right) \\
\text { no disconcordance }
\end{array}
$$

The results of calculation are summarised in Tab. 5 .

Tab. 5 Results of calculation

\begin{tabular}{|c|c|c|c|}
\hline & $\mathbf{a}^{\mathbf{1}}$ & $\mathbf{a}^{\mathbf{2}}$ & $\mathbf{a}^{\mathbf{3}}$ \\
\hline $\mathbf{a}^{\mathbf{1}}$ & $*$ & 0 & 0 \\
\hline $\mathbf{a}^{\mathbf{2}}$ & 0 & $*$ & 0 \\
\hline $\mathbf{a}^{\mathbf{3}}$ & $*$ & 0 & $*$ \\
\hline
\end{tabular}

\begin{tabular}{|c|c|c|c|}
\hline & $\mathbf{a}^{\mathbf{1}}$ & $\mathbf{a}^{\mathbf{2}}$ & $\mathbf{a}^{\mathbf{3}}$ \\
\hline $\mathbf{a}^{\mathbf{1}}$ & $*$ & 0 & 0 \\
\hline $\mathbf{a}^{\mathbf{2}}$ & 0 & $*$ & 0 \\
\hline $\mathbf{a}^{3}$ & $*$ & 0 & $*$ \\
\hline
\end{tabular}

\begin{tabular}{|c|c|c|c|}
\hline & $\mathbf{a}^{\mathbf{1}}$ & $\mathbf{a}^{\mathbf{2}}$ & $\mathbf{a}^{\mathbf{3}}$ \\
\hline $\mathbf{a}^{\mathbf{1}}$ & $*$ & 0 & 0 \\
\hline $\mathbf{a}^{\mathbf{2}}$ & 0 & $*$ & 0 \\
\hline $\mathbf{a}^{\mathbf{3}}$ & $*$ & 0 & $*$ \\
\hline
\end{tabular}

\begin{tabular}{|c|c|c|c|}
\hline & $\mathbf{a}^{\mathbf{1}}$ & $\mathbf{a}^{\mathbf{2}}$ & $\mathbf{a}^{\mathbf{3}}$ \\
\hline $\mathbf{a}^{\mathbf{1}}$ & $*$ & 0 & 0 \\
\hline $\mathbf{a}^{\mathbf{2}}$ & 0 & $*$ & 0 \\
\hline $\mathbf{a}^{3}$ & $*$ & 0 & $*$ \\
\hline
\end{tabular}

\begin{tabular}{|c|c|c|c|}
\hline & $\mathbf{a}^{\mathbf{1}}$ & $\mathbf{a}^{\mathbf{2}}$ & $\mathbf{a}^{\mathbf{3}}$ \\
\hline $\mathbf{a}^{\mathbf{1}}$ & $*$ & 0 & 0 \\
\hline $\mathbf{a}^{\mathbf{2}}$ & 0 & $*$ & 0 \\
\hline $\mathbf{a}^{\mathbf{3}}$ & $*$ & 0 & $*$ \\
\hline
\end{tabular}

\begin{tabular}{|c|c|c|c|}
\hline & $\mathbf{a}^{\mathbf{1}}$ & $\mathbf{a}^{\mathbf{2}}$ & $\mathbf{a}^{\mathbf{3}}$ \\
\hline $\mathbf{a}^{\mathbf{1}}$ & $*$ & 1 & 0 \\
\hline $\mathbf{a}^{\mathbf{2}}$ & 0 & $*$ & 0 \\
\hline $\mathbf{a}^{\mathbf{3}}$ & $*$ & 1 & $*$ \\
\hline
\end{tabular}

Thus, the set of disconcordances $D_{v}$ :

$$
D_{v}=\left\{\left(a^{1}, a^{2}\right),\left(a^{3}, a^{2}\right)\right\}
$$

in the binary form is presented in Tab. 6 .
Tab. 6 Set of disconcordances in the form of binary matrix

\begin{tabular}{|c|c|c|c|}
\hline & $\mathbf{a}^{\mathbf{1}}$ & $\mathbf{a}^{\mathbf{2}}$ & $\mathbf{a}^{\mathbf{3}}$ \\
\hline $\mathbf{a}^{\mathbf{1}}$ & 0 & 1 & 0 \\
\hline $\mathbf{a}^{\mathbf{2}}$ & 0 & 0 & 0 \\
\hline $\mathbf{a}^{\mathbf{3}}$ & 0 & 1 & 0 \\
\hline
\end{tabular}

The set of complements for the set of disconcordances $D_{v}$ is presented in Tab. 7 .

Tab. 7 Set of complements for the set of disconcordances

\begin{tabular}{|c|c|c|c|}
\hline & $\mathbf{a}^{\mathbf{1}}$ & $\mathbf{a}^{\mathbf{2}}$ & $\mathbf{a}^{\mathbf{3}}$ \\
\hline $\mathbf{a}^{\mathbf{1}}$ & 1 & 0 & 1 \\
\hline $\mathbf{a}^{\mathbf{2}}$ & 1 & 1 & 1 \\
\hline $\mathbf{a}^{\mathbf{3}}$ & 1 & 0 & 1 \\
\hline
\end{tabular}

Based on Tab. 4 and Tab. 7, the outranking relations are determined (Tab. 8) - the relation occurs when both the concordance condition (Tab. 4) and the lack of concordance condition (Tab. 7) are met:

$$
S(s, v)=C_{s} \cap D_{v}=\left\{\left(a^{1}, a^{3}\right),\left(a^{2}, a^{1}\right),\left(a^{2}, a^{3}\right)\right\}
$$

Tab. 8 Outranking relation for variants $a^{1}, a^{2}, a^{3}$

\begin{tabular}{|c|c|c|c|}
\hline & $\mathbf{a}^{\mathbf{1}}$ & $\mathbf{a}^{\mathbf{2}}$ & $\mathbf{a}^{\mathbf{3}}$ \\
\hline $\mathbf{a}^{\mathbf{1}}$ & 1 & 0 & 1 \\
\hline $\mathbf{a}^{\mathbf{2}}$ & 1 & 1 & 1 \\
\hline $\mathbf{a}^{\mathbf{3}}$ & 0 & 0 & 1 \\
\hline
\end{tabular}

Graphic image of relationships between variants is presented in the graph in Fig. 1.

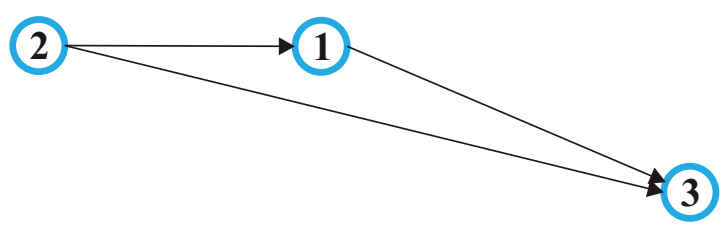

Fig. 1 Graphic image of relationships between variants

Thus, best evaluated is variant no. 2 , i.e. purchase of the suspended diesel railway - variant no. 2 is in outranking relation to both variant no. 1 (purchase of the cog-wheel railway) and variant no. 3 (purchase of the suspended railway with rope drive).

\section{Conclusion}

In the broadly defined management process, among other things the operational research methods 
where the intended objective accomplishment degree is measured by the function of objective (function of criterion) are used very often, and the complexity of the outside world makes the multi-criteria tasks predominate. The Electre I method presented in this article is one of the so-called multi-criteria discrete methods used in relation to the quality features. In this method, the decision maker determines the finite set of variants of decision (in this example, three alternative solutions for purchase of the means of transport are taken into consideration) and the finite set of criteria (in this case, comprised of 6 elements), which allows generating a single variant that best corresponds to its preferences.

\section{References}

HILLIER, F. S., LIEBERMAN, G. J. (1990). Introduction to Operations Research. Mc Graw - Hill, 1990.

MYNARSKI, S. (1992). Badania przestrzenne rynku i konsumpcji. Przewodnik metodyczny. Wydawnictwo Naukowe PWN, Warszawa, 1992.

TRZASKALIK, T. (2006). Metody wielokryterialne na polskim rynku finansowym. PWE, Warszawa, 2006.

TRZASKALIK, T. (2001). Modelowanie optymalizacyjne. Absolwent, Łódź, 2001.

WAGNER, H. (1980). Badania operacyjne. zastosowania w zarzadzaniu. PWE, Warszawa, 1980. 